Nonlinear Processes in Geophysics (2003) 10: 397-405

Nonlinear Processes

in Geophysics

(C) European Geosciences Union 2003

\title{
Development of internal solitary waves in various thermocline regimes - a multi-modal approach
}

\author{
T. Gerkema \\ Royal Netherlands Institute for Sea Research, P.O. Box 59, 1790 AB Den Burg, The Netherlands
}

Received: 17 September 2002 - Revised: 5 November 2002 - Accepted: 6 November 2002

\begin{abstract}
A numerical analysis is made on the appearance of oceanic internal solitary waves in a multi-modal setting. This is done for observed profiles of stratification from the Sulu Sea and the Bay of Biscay, in which thermocline motion is dominated by the first and third mode, respectively. The results show that persistent solitary waves occur only in the former case, in accordance with the observations. In the Bay of Biscay much energy is transferred from the third mode to lower modes, implying that a uni-modal approach would not have been appropriate. To elaborate on these results in a systematic way, a simple model for the stratification is used; an interpretation is given in terms of regimes of thermocline strength.
\end{abstract}

\section{Introduction}

Internal solitary waves in the ocean have been conceived predominantly from the viewpoint of interfacial waves, see e.g. the mechanisms for their generation as put forward by Maxworthy (1979), Osborne and Burch (1980) and Apel et al. (1985). Even if the notion of continuous stratification was adopted (involving, in principle, a set of vertical modes), then in semi-analytical studies still only one mode was selected (e.g. Lee and Beardsley, 1974; Liu et al., 1985; New and Pingree, 2000). Such a restriction is attractive because it allows one to reduce the equations to simple evolution equations like Korteweg-de Vries (KdV) equation or kindred soliton equations. This uni-modal approach rests on the (tacit) assumption that the selected mode would not be disturbed if other modes were present, i.e. would not be susceptible to inter-modal interaction. The main purpose of this paper is to delineate the conditions under which this assumption can be expected to be valid.

In purely numerical studies on solitary waves there is no need to make a uni-modal assumption. For example in the model developed and used by Lamb (1994), which allows

Correspondence to: T. Gerkema (gerk@nioz.nl) for a continuous stratification, many modes are implicitly taken into account. Yet, if possible, it is attractive to interpret the model results by making a connection with simple and well-understood analytical theory (like KdV); thus it is important to know under what conditions a simple (uni-modal) approach is justifiable.

On the observational side, two contrasting types of behaviour have been described in the literature. Internal solitary waves may show a remarkable longevity, like in the Sulu Sea (Apel et al., 1985), where they travel distances of over $450 \mathrm{~km}$, until they reach the coast of Palawan Island, where they still are energetical enough to create considerable seiches in Puerto Princesa harbor (Giese et al., 1998). In this case a uni-modal approach seems appropriate: the solitary waves are well described by the first mode alone, which here has its maximum near the thermocline, giving the mode an interfacial character. The whole process of generation and propagation can indeed be described entirely in terms of this "interfacial" mode (Liu et al., 1985; Gerkema, 1994).

The behaviour of internal solitary waves in the Bay of Biscay offers a remarkable contrast, both in their generation and propagation. The generation process, the observational evidence of which is described by New and Pingree (1990, 1992); New and Da Silva (2002), is now well-understood; the initial thermocline disturbance from which the solitary waves emerge is provoked by scattering of an incoming internaltide beam at the thermocline (Gerkema, 2001), the scattering itself being an essentially linear process. It was also found that the solitary waves decay fairly rapidly (New and Pingree, 1992), which is quite unlike the observations in the Sulu Sea. The very fact that an internal-tide beam is crucial renders a uni-modal approach inadequate (since the description of a beam requires a number of modes to be included). Yet if one would start the analysis by regarding the thermocline disturbance as given, then its later evolution would possibly (but not necessarily) be well represented by one single mode (in this case: the third mode, see Sect. 3). This approach was followed by New and Pingree (2000); here we will explore to what extent it can be justified. 
In a previous study (Gerkema, 2001), the generation process in the Bay of Biscay was studied with a simple nonlinear nonhydrostatic internal-tide generation model, using 1215 modes. No explicit analysis was made of energy transfer between modes, as we will do here; to this end we study the evolution of a given initial profile (instead of incorporating a full generation mechanism), and take into account the main modes. First a brief description of the model is given (Sect. 2), with an additional discussion on the formulation of energy-conservation. Then we apply the model to the Sulu Sea and Bay of Biscay, using the observed profiles of the buoyancy frequency (Sect. 3). Finally, we use a simple model for the stratification ("2c-layer") to study the differences found in the previous section in a systematic way, in terms of stratification regimes, measured by the strength of the thermocline.

\section{Model equations}

The model used here includes nonlinear and nonhydrostatic effects, but is frictionless, and uniform in one of the horizontal directions $(y)$. Coriolis effects will be ignored here (except at one instance in Sect. 3); they are already known to have an adverse effect on the generation of internal solitary waves (Gerkema and Zimmerman, 1995; Gerkema, 1996). Here we want to focus purely on the transfer of energy between modes, in the absence of other complicating factors. The variables to be solved are the baroclinic streamfunction $\psi\left(u=\psi_{z}, w=-\psi_{x}\right)$ and $\rho$, which is the density perturbation with respect to its local static value (multiplied by $g / \rho_{*}$; $g$ the acceleration due to gravity, $\rho_{*}$ a reference value for density). The equation expressing mass conservation $(\nabla \cdot \boldsymbol{u}=0)$ being automatically satisfied due to the streamfunction formulation, the remaining equations are the momentum equations for $u$ and $w$, combined into one equation for vorticity $\left(\nabla^{2} \psi\right)$, and the equation expressing energy conservation:

$$
\begin{gathered}
\nabla^{2} \psi_{t}+J\left(\nabla^{2} \psi, \psi\right)-\rho_{x}=0 \\
\rho_{t}+J(\rho, \psi)+N^{2} \psi_{x}=0
\end{gathered}
$$

with $\nabla^{2}=\partial^{2} / \partial x^{2}+\partial^{2} / \partial z^{2}$; the Jacobians are defined by $J(a, b)=a_{x} b_{z}-a_{z} b_{x} ; N$ the buoyancy frequency, here assumed to be a function of the vertical coordinate $z$ only. In contrast to Gerkema (2001), no forcing is included; here we will study the evolution of a given initial depression.

\subsection{Multi-modal approach}

The vertical modes $\phi_{n}$, associated with the linear hydrostatic version of Eqs. (1,2), satisfy

$\phi_{n}^{\prime \prime}+\frac{N^{2}}{c_{n}^{2}} \phi_{n}=0$

(denoting derivatives in $z$ by primes) along with the boundary conditions $\phi_{n}=0$ at $z=0,-H$, i.e. at surface and bottom; $c_{n}$ is the phase-speed of the $n$th mode.
We shall assume that nonlinear and nonhydrostatic effects are weak in Eqs. $(1,2)$, and of equal order of strength. This implies that results from the linear hydrostatic equations may be used to simplify the nonlinear and dispersive terms, and that combined effects of nonlinearity and dispersion (the $\psi_{x x}$ in the Jacobian in Eq. 1) can be neglected. We expand the fields in terms of the vertical modes $\phi_{n}$ :

$$
\psi=\sum_{n=1}^{\infty} a_{n}(t, x) \phi_{n}(z) ; \quad \rho=\sum_{n=1}^{\infty} N^{2} b_{n}(t, x) \phi_{n}(z) .
$$

As was shown in detail in Gerkema (2001), using orthogonality of the modes (and introducing a correction term in the modal expansion), finally leads to the following set of equations for the modal coefficients:

$$
\begin{aligned}
& a_{k, t}-c_{k}^{2} a_{k, x x t} R_{k}+c_{k}^{2} \sum_{n, m} a_{n, x} a_{m} \\
& \left\{S_{k m n}\left(\frac{1}{c_{n}^{2}}-\frac{1}{c_{m}^{2}}\right)-T_{k m n} \frac{1}{c_{m}^{2}}\right\}+c_{k}^{2} b_{k, x}=0 \\
& b_{k, t}+\sum_{n, m} \\
& \left\{\left(b_{n, x} a_{m}-a_{n, x} b_{m}\right) S_{k m n}-a_{n, x} b_{m} T_{k m n}\right\}+a_{k, x}=0,
\end{aligned}
$$

where

$$
\begin{aligned}
& P_{k}=\int d z N^{2} \phi_{k}^{2} ; \quad R_{k}=\frac{\int d z \phi_{k}^{2}}{P_{k}} ; \\
& S_{k m n}=\frac{\int d z N^{2} \phi_{k} \phi_{m}^{\prime} \phi_{n}}{P_{k}} ; \quad T_{k m n}=\frac{\int d z\left(N^{2}\right)^{\prime} \phi_{k} \phi_{m} \phi_{n}}{P_{k}} .
\end{aligned}
$$

Equations (4) and (5) serve as a starting point for the analysis in the rest of this paper.

\subsection{The connection between nonlinearity and stratification}

Here we discuss briefly under what conditions nonlinearity can play a role at all, recapitulating some known results (LeBlond and Mysak, 1978; Ostrovsky, 1978).

First we consider the case of constant stratification $(N=$ $N_{c}$ ). As is well-known (LeBlond and Mysak, 1978), in an unbounded medium a monochromatic beam $\psi, \rho \sim \exp i(k x+$ $m z-\sigma t)$ forms a solution of the nonlinear Eqs. $(1,2)$, provided that the pair $(\sigma, m / k)$ satisfies the dispersion relation. This is because all Jacobians vanish in this case. (Notice that to arrive at Eq. (1) the Boussinesq approximation was made; without this approximation nonlinearities of a different type would arise that are not zero, see Benney, 1966.) A superposition of beams of different frequencies (each of which separately satisfying (Eqs. 1, 2)) does not provide a solution of Eqs. $(1,2)$; this property was exploited in Shrira (1981) to derive an amplitude equation for a (three-dimensional) internalwave packet with a narrow-band spectrum.

In systems bounded below and above (flat bottom, rigidlid), the propagation of a monochromatic beam involves reflections at the boundaries, giving superpositions of incoming and outgoing beams, and non-vanishing nonlinear terms. 
This last point can be seen as follows: in such a bounded system internal-wave propagation can be described alternatively by vertical modes, and as the multi-modal set (Eqs. 4, 5) indicates, nonlinearities are always present, even for constant $N$, in which case:

$T_{k m n}=0 ; \quad S_{k m n}=\frac{N_{c}}{2 c_{m}} \times \begin{cases}-1 & \text { if } m=|k+n| \\ +1 & \text { if } m=|k-n| \\ 0 & \text { else }\end{cases}$

$T$ vanishes because of constant $N$, but $S$ does not as long as more than one mode is present. It is only if we reduce the set (artificially) to one mode that nonlinearity disappears altogether from Eqs. $(4,5)$.

Importantly, for non-constant $N$ nonlinear terms do not disappear (not even in a uni-modal approach). In this sense, one might say that the thermocline forms a major "source" of nonlinearity, not only locally, but for the whole watercolumn.

\subsection{Conservation of energy}

In the following sections we will investigate (numerically) the transfer of energy between modes. Of course, we must check that the total energy remains constant. This entails a complication, for it turns out that the usual definition of internal-wave energy-density

$$
\frac{1}{2}\left(\psi_{x}^{2}+\psi_{z}^{2}+\frac{\rho^{2}}{N^{2}}\right)
$$

fails; this is because we consider nonlinear waves in a nonconstantly stratified medium. (As will become clear below, Eq. (7) represents energy-density only if one of the "nons" were skipped.) A correction to Eq. (7) can be found in Miropol'sky (2001); however, only a sketch of the derivation is given there, involving a Hamiltonian formulation. As an alternative, we here provide a rather more detailed and elementary derivation, with some additional remarks.

It can be easily derived from Eqs. $(1,2)$ that the quantity

$$
\frac{1}{2}\left(\psi_{x}^{2}+\psi_{z}^{2}\right)+z \rho
$$

is conserved (i.e. its time derivative plus the divergence of a flux equals zero). The expression remains conserved if one adds the (time-independent) term $z \rho_{0}$, where $\rho_{0}(z)$ is the static density; this gives the usual expression for kinetic plus potential energy. Unfortunately, Eq. (8) does not provide a suitable starting point for the calculation of energy in terms of modes. This can be seen by considering, for example, an initial profile of a mode given by $\rho=N^{2} \sin x \phi_{n}(z)$, which, if substituted in $z \rho$ and integrated over one horizontal wavelength, appears to contain as much energy as does $\rho=0$, which of course must be false. (Recall that $\rho$ does not denote density itself but the density perturbation due to wave-motion.) The underlying cause is that the modal series as such represent infinitesimal displacements, and we should first make explicit the nonlinear effects implicitly contained in $z \rho$ before we insert the modal expressions (thus spoiling, however, the exactness). This is most easily done by returning to Eq. (1), which after multiplication by $\psi$ and rearranging of terms becomes:

$$
\begin{aligned}
& -\frac{1}{2}\left[\psi_{x}^{2}+\psi_{z}^{2}\right]_{t}+\left[\psi \psi_{x t}\right]_{x}+\left[\psi \psi_{z t}\right]_{z} \\
& +\left[\left(\nabla^{2} \psi\right) \psi \psi_{z}\right]_{x}-\left[\left(\nabla^{2} \psi\right) \psi \psi_{x}\right]_{z}-\psi \rho_{x}=0 .
\end{aligned}
$$

We rewrite the last term as $-[\psi \rho]_{x}+\psi_{x} \rho$, in which the second term $(-w \rho)$ can be reduced by expressing $w$ in terms of isopycnal displacements $\eta$, and then expressing $\eta$ in terms of $\rho$. In the first step we use the condition for a material interface

$w(t, x, z+\eta)=\eta_{t}(t, x, z)+u(t, x, z+\eta) \eta_{x}(t, x, z)$,

where $\eta(t, x, z)$ denotes the isopycnal displacement of the surface that would lie at depth $z$ if the fluid were at rest. It is important here to denote the arguments explicitly, since what we need is $w$ at depth $z$ (instead of at $z+\eta$ ), so we must develop Eq. (10) in a Taylor expansion, the first few terms of which are

$w+w_{z} \eta+\cdots=\eta_{t}+u \eta_{x}+\cdots$,

where $w$ and $u$ are now evaluated at depth $z$. At lowest order this gives $w=\eta_{t}$, and hence at the next order we find

$w=\eta_{t}+u \eta_{x}-\eta \eta_{t z}+\cdots$.

A connection between $\eta$ and $\rho$ can be obtained from the identity $\bar{\rho}(t, x, z+\eta)=\rho_{0}(z)$, which holds for incompressible fluids (here $\rho_{0}$ denotes the static density distribution; $\rho_{0}, \rho$ and $\bar{\rho}=\rho_{0}+\rho$ have been multiplied by $g / \rho_{*}$ for convenience, hence $\left.N^{2}=-\rho_{0, z}\right)$. Developing the identity in a Taylor expansion around $z$, we obtain

$\rho_{0}+\rho_{0, z} \eta+\frac{1}{2} \rho_{0, z z} \eta^{2}+\cdots+\rho+\rho_{z} \eta+\cdots=\rho_{0}$,

in which the quantities are now evaluated at $z$. Hence

$-N^{2} \eta-\frac{1}{2}\left(N^{2}\right)_{z} \eta^{2}+\rho+\rho_{z} \eta+\cdots=0$.

At lowest order this gives $\rho=N^{2} \eta$, and using this result in the quadratic terms we find, at next order,

$N^{2} \eta=\rho+\rho_{z} \frac{\rho}{N^{2}}-\frac{1}{2}\left(N^{2}\right)_{z}\left(\frac{\rho}{N^{2}}\right)^{2}+\cdots$.

Using Eq. (11) and Eq. (12), and neglecting terms of orders higher than cubic, we finally obtain

$w \rho=\frac{1}{2}\left(\frac{\rho^{2}}{N^{2}}\right)_{t}+\frac{1}{6}\left(N^{2}\right)_{z}\left(\frac{\rho^{3}}{N^{6}}\right)_{t}+\frac{1}{2}\left[\psi_{z} \frac{\rho^{2}}{N^{2}}\right]_{x}+\frac{1}{2}\left[\frac{\rho^{2} \rho_{t}}{N^{4}}\right]_{z}$,

in which the last two terms are in conservative form. Hence we find from Eq. (9) that the expression

$\frac{1}{2}\left(\psi_{x}^{2}+\psi_{z}^{2}+\frac{\rho^{2}}{N^{2}}+\frac{1}{3} \frac{\rho^{3}}{N^{6}}\left(N^{2}\right)_{z}\right)$

represents a conserved quantity if we neglect nonlinear terms higher than cubic; this is the correction to Eq. (7). 

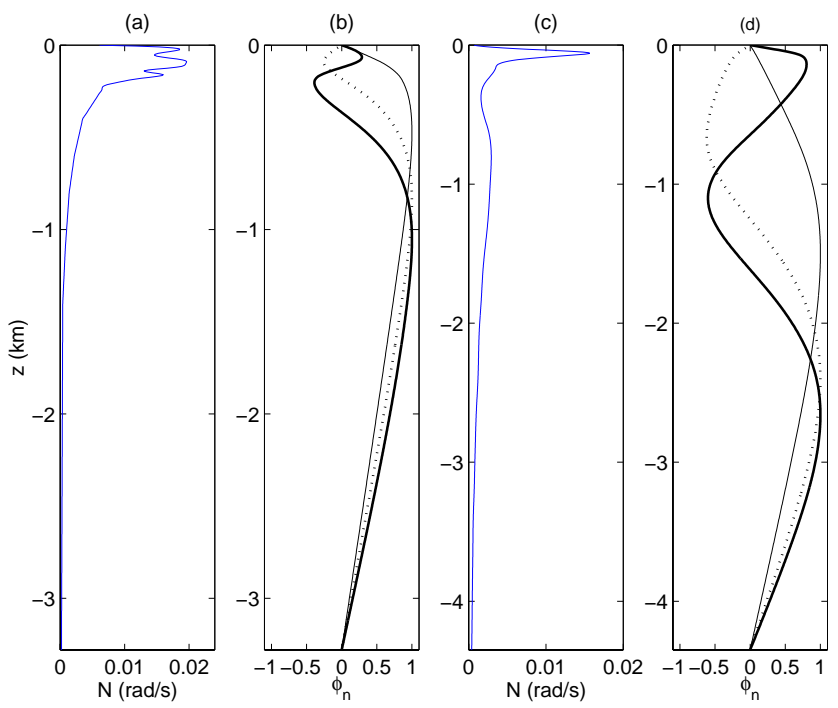

Fig. 1. The profiles of $N$ for the Sulu Sea (a), and the Bay of Biscay (c), after Apel et al. (1985) and Pingree and New (1991), respectively. The corresponding modal structures ((b) and (d)) have been obtained by solving Eq. (3); the first, second and third modes are indicated by thin, dotted and thick lines, respectively.

Henceforth we will use Eq. (14); although still not an exactly conserved quantity, it suffices for our purposes. Higherorder terms could be obtained if needed, either by the procedure we used above, or by the one described by Miropol'sky (2001).

Finally, we emphasize again the importance of writing down the arguments in Eq. (10); their omission may cause confusion. For example, the conservation law in Apel (1987, Eq. 4.70), in which the potential energy is described by $\rho \eta$ (in our notation), has the appearance of being exact, whereas in fact it is valid only at lowest order (to obtain it use was made of Eq. (10), but since the arguments are omitted it is no longer clear that the derivation involves an approximation).

\subsection{2c-layer stratification}

In Sect. 4 we shall use a "2c-layer" model (Baines, 1982; Gerkema, 2001): it consists of an unstratified upper layer, a constantly stratified lower layer, and a jump in density across the interface, which represents the thermocline. Thus:

$N^{2}(z)=g^{\prime} \delta(z+d)+N_{c}^{2} \Theta(-z-d)$.

Here $\Theta$ is the Heaviside-step function (being 1 for positive argument and zero elsewhere); $\delta$ the delta-distribution; $g^{\prime}$ is reduced gravity, i.e. the acceleration $g$ multiplied by the relative difference in density across the interface; $d$ denotes the depth of the thermocline (interface).

For this $N(z)$, the eigenvalue problem (Eq. 3) yields

$$
\begin{aligned}
& \phi_{n}(z)= \begin{cases}-\frac{z}{d} \sin q_{n}(H-d) & -d<z<0 \\
\sin q_{n}(z+H) & -H<z<-d .\end{cases} \\
& q_{n} d=\left(\frac{g^{\prime} d}{c_{n}^{2}}-1\right) \tan q_{n}(H-d)
\end{aligned}
$$

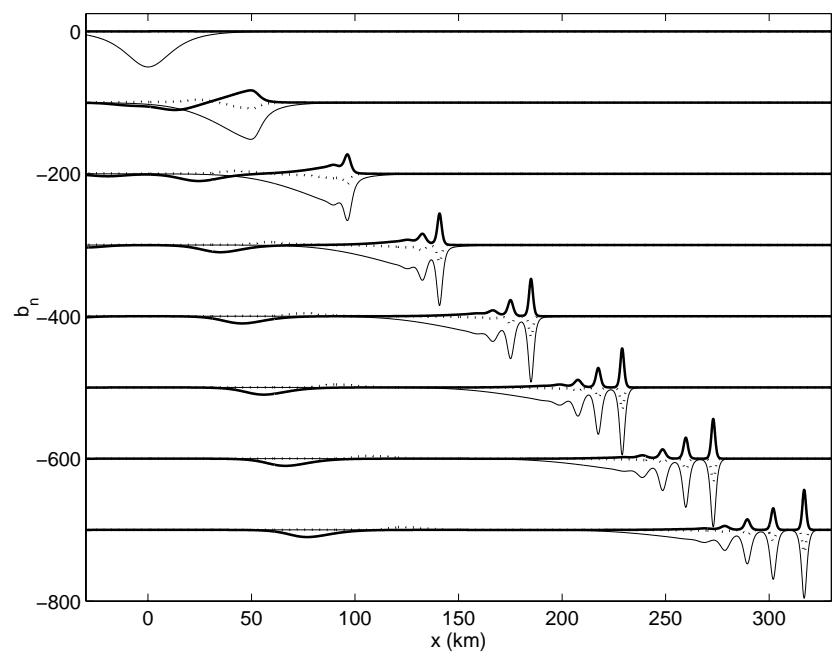

Fig. 2. The evolution of an initial first-mode depression, for a stratification as in Sulu Sea. A train of solitary waves emerges, and in each of them the higher modes give a small contribution. Modes indicated as in the previous figure. Time-lapse between subsequent stages is $4.5 \mathrm{~h}$.

in which $q_{n}=N_{c} / c_{n}$. The eigenvalues $c_{n}$ can be solved numerically from the dispersion relation (Eq. 17) for given parameters $d, H, g^{\prime}$ and $N_{c}$. For this stratification the integral expressions for $P_{k}$ etc. are given in analytical form in Gerkema (2001), and will not be reproduced here.

\section{Sulu Sea versus Bay of Biscay}

In this section we elaborate on the distinction between the Sulu Sea and the Bay of Biscay, as regards the behaviour of internal solitary waves. A distinctive feature can immediately be seen in the modal structures, shown in Fig. 1 (note: here and throughout the rest of this paper, we use thin solid lines for the first mode, dotted lines for the second mode, and thick solid lines for the third mode). Whereas in the Sulu Sea the first mode has its maximum at the base of the thermocline, in the Bay of Biscay the third mode has a (local) maximum at the thermocline. Hence we expect an initial depression of the thermocline in these two regions to be dominated by the first mode and the third mode, respectively; this is in line with earlier uni-modal numerical studies (Liu et al., 1985; Gerkema, 1994; New and Pingree, 2000, the first two dealing with the Sulu Sea, the third with the Bay of Biscay). Moreover, in the Bay of Biscay the dominance of the third mode was confirmed observationally, see New (1988).

Here we will investigate whether a uni-modal approach can be justified in either of the cases. In solving the multimodal set (Eqs. 4, 5), we take into account the first three modes. A finite-difference method was used: a 3-point symmetric formula for the time-derivative (and also for the second derivative in $x$ ), and a 5-point symmetric formula for the horizontal derivative (see Fletcher, 1991). The coefficients 


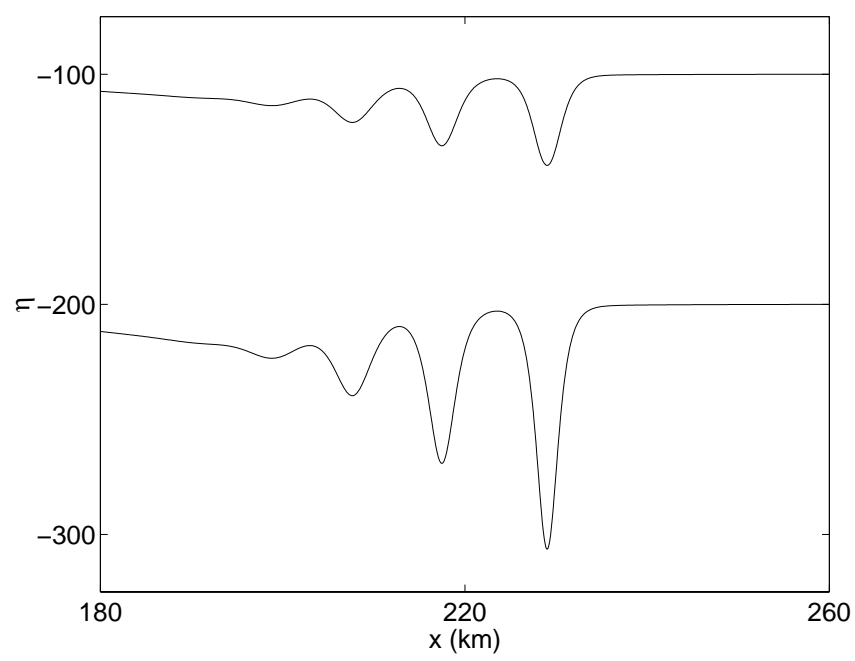

Fig. 3. The isothermal displacements at two different depths (Sulu Sea); due to the multi-modal character of the signal, the profiles are dissimilar.

$P_{k}, R_{k}, S_{k m n}$ and $T_{k m n}$, which appear in the equations, are evaluated numerically beforehand. We present the results by depicting the modal coefficients $b(t, x)$; thus we implicitly also visualize the isopycnal displacements $\eta$ at any depth, because

$\eta=\sum_{n=1}^{3} b_{n}(t, x) \phi_{n}(z)$

where $\phi_{n}$ is the vertical modal structure shown in Fig. 1.

For the Sulu Sea, we take as an initial profile a first-mode depression, choosing one of (roughly) similar size and proportion as in Liu et al. (1985). To make it propagate to the right, we choose $a_{1}=c_{1} b_{1}$, initially. In the finite-difference scheme steps were $\Delta x=100 \mathrm{~m}$ and $\Delta t=10 \mathrm{~s}$. Figure 2 shows the result of the model: the depression develops neatly into a sequence of amplitude-ordered solitary waves. In each of them, the first-mode structure is accompanied by a similar but smaller third-mode structure, and an even smaller second-mode one (the former appears in Fig. 2 as an elevation, the latter as a depression). In other words, during the evolution energy has been transferred from the first mode to higher ones; the amount involved is small: less than $2 \%$.

However, locally there may be noticeable effects. For example in $\eta$ (given by Eq. 18) the ratio of amplitudes of consecutive solitary waves is substantially different at different depths (in a uni-modal analysis the ratio would be the same for all depths), as can be clearly seen in Fig. 3; this is in qualitative agreement with the observations (Apel et al., 1985). Put into quantitative terms, the difference is as follows: at $200 \mathrm{~m}$ depth the ratio of the first solitary wave to the second is 1.54 , and of the first to the third 2.68; at $100 \mathrm{~m}$ depth these ratios are smaller: 1.27 and 1.89 .

Apart from these effects, we can conclude (given the small amount of energy-transfer) that a uni-modal approach, i.e.

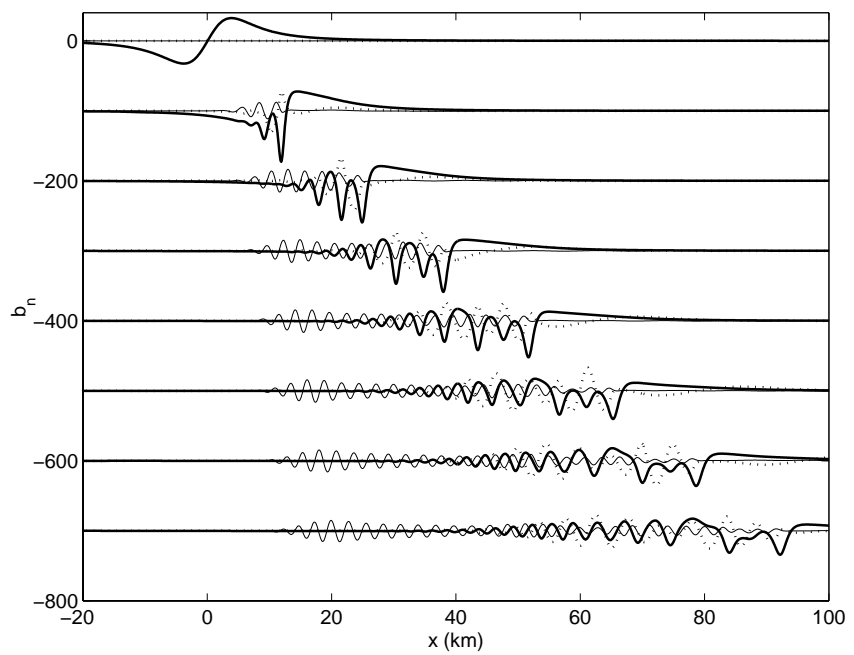

Fig. 4. The evolution of an initial third-mode depression, for a stratification as in the Bay of Biscay. Initially, a train of solitary waves develops, but soon crumbles, while the other modes participate strongly. Time-lapse between subsequent stages is $4.5 \mathrm{~h}$.

ignoring modes higher than the first, would have been fairly reasonable, at least as a first approximation.

We now turn to the Bay of Biscay, where (for the abovementioned reasons) the initial profile will be chosen to consist of the third-mode alone, with $a_{3}=c_{3} b_{3}$. Here we choose an initial profile whose integral is zero, because Coriolis effects can then be incorporated in a natural way (for a single depression the transverse velocity component would remain finite far away from the depression).

The evolution according to Eqs. $(4,5)$, for the first three modes, is shown in Fig. 4, here without Coriolis effects. At the early stages of development a train of solitary waves seems to emerge, but the pattern soon becomes irregular and the peaks ("solitary waves") generally become smaller; also, the first two modes soon play a prominent part. Evidence of the latter feature is more clearly seen in the distribution in time of energy-density, given by Eq. (14), see Fig. 5: almost half of the energy originally contained in the third mode leaks away to the first two modes.

In contrast to the Sulu Sea, which is located very close to the equator, in the Bay of Biscay Coriolis effects are not negligible in internal-tide and solitary-wave dynamics. Hence we repeated the previous calculation, now taking Coriolis effects into account $\left(f=1 \cdot 10^{-4} \mathrm{~s}^{-1}\right)$. This requires an adaptation of Eqs. $(4,5)$, see Gerkema (2001). In this case (not shown) the solitary waves are less prominent, and in any case much less form-preserving than in the previous case. The energy transferred from the third mode to the first two is still significant, though smaller: being about one-quarter. All in all, the qualitative conclusions are the same as without Coriolis effects.

To summarize, in a multi-modal analysis a clear contrast is seen between the evolution of the "thermocline modes" in the Sulu Sea and Bay of Biscay (first and third mode, respec- 


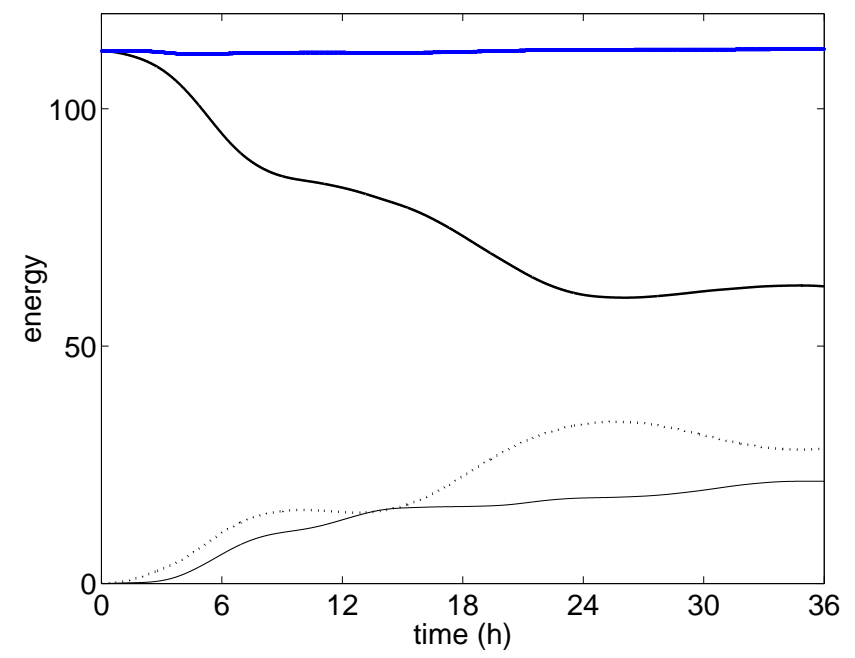

Fig. 5. The time-development of energy-density for each of the participating modes; the upper line represents the total energy.

tively; other choices will be considered in the next section). Whereas in the Sulu Sea the first-mode depression develops into a well-defined train of persistent solitary waves with no significant loss of energy to other modes, in the Bay of Biscay the pattern is much more blurred, the solitary waves less steady, and much energy is transferred to the first two modes. In the next section we will generalize these results by pinpointing the essential difference between the two cases: the thermocline strength.

\section{Classification in terms of 2c-layer model}

In this section we use the 2c-layer stratification model, see Eq. (15), which despite its simplicity captures the two key elements of the oceanic stratification: the presence of a thermocline and a weak abyssal stratification. The model is governed by only four parameters, three of which will be kept fixed: $d=50 \mathrm{~m}$ (thermocline depth), $H=4000 \mathrm{~m}$ (waterdepth), $N_{c}=0.002 \mathrm{~s}^{-1}$ (abyssal stratification). Only $g^{\prime}$, the thermocline strength, is varied (units are $\mathrm{m} \mathrm{s}^{-2}$ - for brevity we will not repeat them below; for a simple geometric interpretation of $g^{\prime}$, see Gerkema, 2001).

Following Baines (1982) and Gerkema (2001), we can distinguish three regimes $\mathrm{A}, \mathrm{B}$, and $\mathrm{C}$, in which the thermocline is weak, strong, and moderate, respectively. This corresponds to a distinction in modal structures, see Fig. 6: in A, the modal patterns are very similar to those in a constantly stratified fluid (i.e. sinusoidal); in B, the first mode has its maximum near the thermocline (much like an interfacial mode in a two-layer system); finally, in a range of intermediate values $(\mathrm{C})$, the third mode has an extremum near the thermocline, while the first and second modes look much like those in A. (There is of course a smooth transition of the modal structures from one regime to another, but here we will focus on the distinctive ones shown in Fig. 6.) The connec-
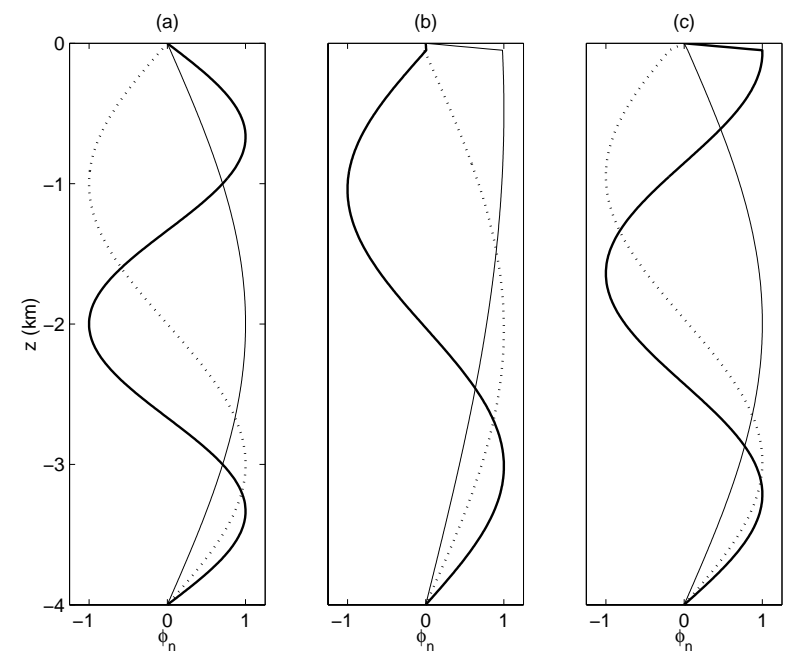

Fig. 6. Modal structures for the 2c-layer model, defined by (15), for three typical stratification regimes, in which the thermocline is: weak or absent $\left((\mathbf{a}), g^{\prime}=0\right)$; very strong $\left((\mathbf{b}), g^{\prime}=0.4\right)$; and moderate $\left((\mathbf{c}), g^{\prime}=0.02\right)$. Recall that the first, second and third modes are indicated by thin, dotted and thick lines, respectively.

tion with the previous section is that Fig. $6 \mathrm{~b}$ is reminiscent of Fig. 1b, and Fig. $6 c$ of Fig. 1d.

By varying $g^{\prime}$ we also vary the quantity

$\gamma=\frac{\left(g^{\prime} d\right)^{1 / 2}}{N_{c} H}$,

which in fact is the determining factor in distinguishing the regimes (see Gerkema, 2001); this shows how we would go from one regime to another if for instance $d$ were varied instead of $g^{\prime}$. As before, quantities referring to the first, second and third mode will be plotted in thin lines, dotted lines, and thick lines, respectively.

\subsection{Development of first-mode depression}

In this section we study the development of a first-mode depression $b_{1}$, with $a_{1}=c_{1} b_{1}$ (initially) which makes the depression move to the right. We consider each of the three regimes of stratification indicated in Fig. 6, but notice that the first-mode depression can be associated with a distinct thermocline depression only in regime B. In all cases the first three modes are taken into account.

For weak and moderate regimes (i.e. A and C) no solitons emerge and the profile develops only a dispersive tail (not shown), which is due to the dynamics of the first mode itself; virtually no energy is transferred to the higher modes. Only for sufficiently strong thermoclines, regime $\mathrm{B}$, do solitons emerge, see Fig. 7. Associated with each soliton is a small elevation of the third mode (and to a lesser extent, of the second mode too), but the contribution to the interfacial (thermocline) excursion remains negligible because of the small local values of $\phi_{3}$ and $\phi_{2}$.

For all three values of $g^{\prime}$ the amount of energy transferred from the first mode to higher modes is insignificant, be- 


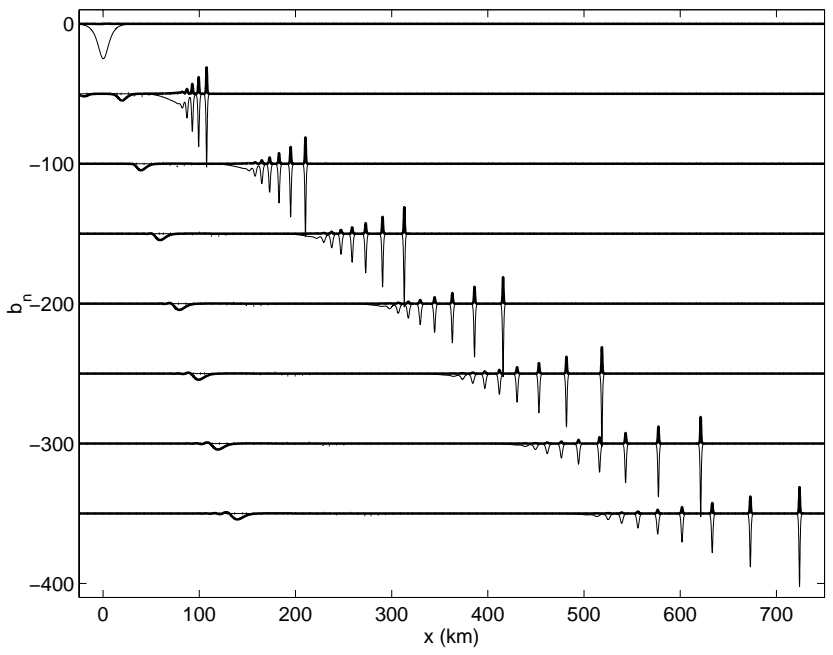

Fig. 7. The development of an initial first-mode depression in regime $\mathrm{B}$ (strong thermocline). Time-lapse between subsequent stages is $4.5 \mathrm{~h}$.

ing less than $0.1 \%$; in other words, a uni-modal approach would have been appropriate here. Notice that we deal here only with a "free" exchange of energy; the presence of topographic features may lead to a different outcome, as was shown numerically and in laboratory experiments by Vlasenko and Hutter (2001), where a first-mode profile, when encountering a sill, lost much energy to the second mode.

\subsection{Development of third-mode depression}

We now study the development of a third-mode depression $b_{3}$, with $a_{3}=c_{3} b_{3}$ (initially) which makes the depression move to the right. We consider each of the three regimes of stratification indicated in Fig. 6, but notice that the thirdmode depression can be associated with a thermocline depression only in regime $\mathrm{C}$. Furthermore, in none of the cases does the profile manifest itself as a depression throughout the water column; the change of sign in $\phi_{3}$ turns it into an elevation in part of the column.

For comparison, we first show the development of the third mode in a uni-modal approach, here for $g^{\prime}=0.02$ (regime $\mathrm{C}$ ), see Fig. 8: the depression evolves into a pattern of amplitude-ordered solitary waves, like in common soliton equations (e.g. $\mathrm{KdV}$ ).

Since it is rather artificial to exclude other modes from taking part, we now allow the first and second modes to interact with the third, see Fig. 9. These modes are seen to develop oscillations at the expense of the third mode. In particular, the first mode manifests itself as a group of high-frequency waves, which will be most prominent half-way down the watercolumn, because the first mode has its maximum there. This, apparently, is the origin of the oscillations noticed in the more complicated context of earlier numerical experiments (Gerkema, 2001). A conspicuous feature in Fig. 9 is a "twin-type" solitary wave, which propagates steadily apart

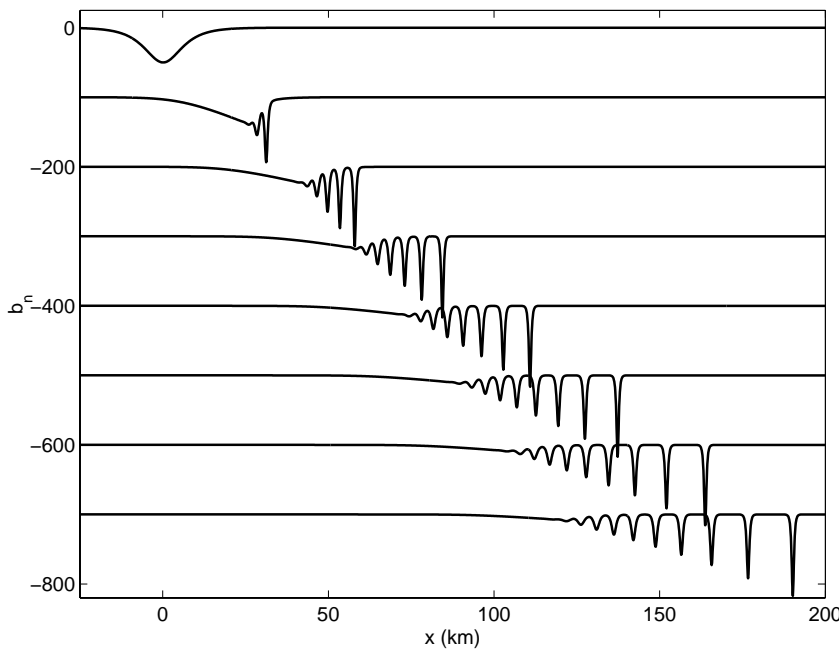

Fig. 8. Development of initial third-mode depression when all other modes are excluded (uni-modal approach), for regime $\mathrm{C}$ (moderate thermocline). Time-lapse between subsequent stages is $4.5 \mathrm{~h}$.

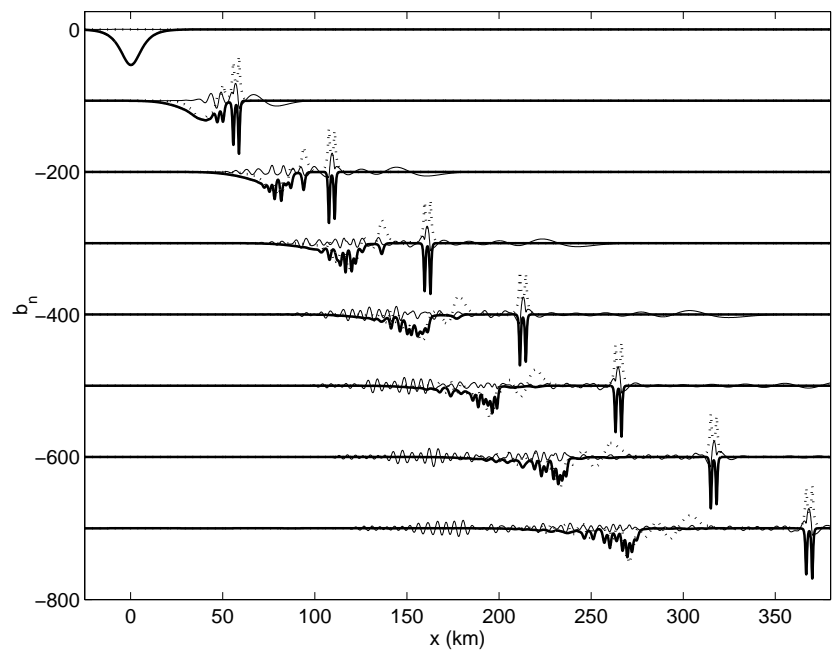

Fig. 9. Development of initial third-mode depression when the first and second mode are taken into account too, again for regime $\mathrm{C}$ (moderate thermocline). Time-lapse between subsequent stages is $9 \mathrm{~h}$.

from a small but persistent oscillation. Although the first mode seems least important here, it turns out that it plays a crucial role in keeping the "twins" together (this was checked by performing a calculation with the second and third mode alone). Interestingly, "twin" patterns have indeed been observed in the Bay of Biscay (New and Pingree, 1990). The possibility to describe this pattern analytically by Eqs. $(4,5)$ (or a simplified version of it) will not be probed here. To our knowledge, the only analytical description of multi-modal solitons was made by Vlasenko (1994), but this involved a correction to the known uni-modal KdV soliton (square hyperbolic secans; the paper states $\operatorname{coth}^{-2}$, an apparent misprint), rather than an entirely new structure.

The appearance of a multi-modal twin-type solitary wave 


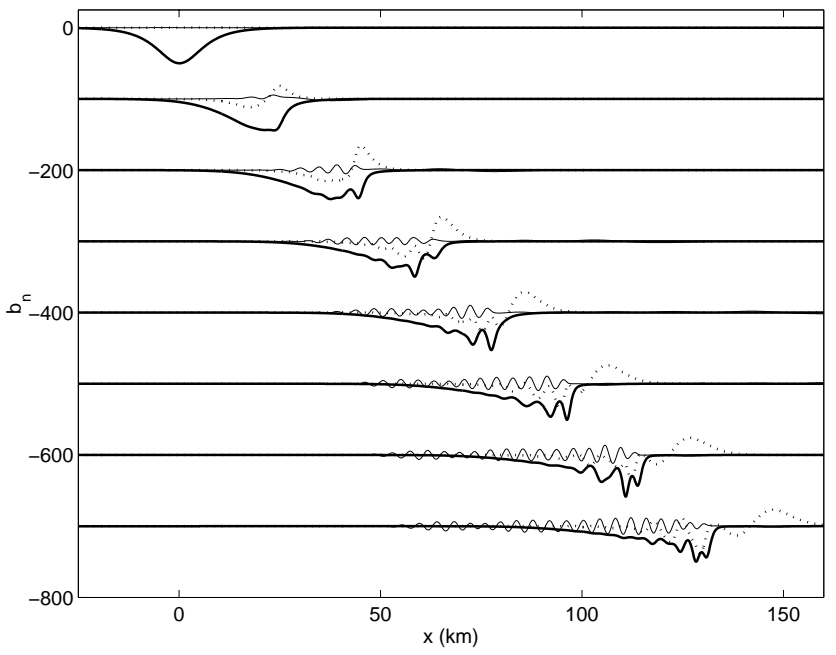

Fig. 10. Same as in Fig. 9, but for a slightly weaker thermocline $\left(g^{\prime}=0.015\right)$. Time-lapse between subsequent stages is $4.5 \mathrm{~h}$.

as shown in Fig. 9 is not typical of regime C, since it appears only in a small range of $g^{\prime}$. For example for a somewhat smaller value $g^{\prime}=0.015$ the pattern is entirely absent, see Fig. 10. Here solitary waves, if present at all, are certainly not well-defined. Like in Fig. 9, the first mode manifests itself as groups of modulated high-frequency waves.

For very weak or very strong thermoclines (not shown), i.e. regimes $\mathrm{A}$ and $\mathrm{B}$, we find that the depression undergoes only a minor distortion and does not develop into solitary waves. Also, virtually no energy is transferred to other modes. For the whole range of thermocline regimes, the results are collected in Fig. 11; we see indeed that the third mode loses energy only for moderate values of thermocline strength, i.e. regime $\mathrm{C}$.

Finally, it has been checked whether the above results are sensitive to the inclusion of even more modes (i.e. fourth and higher). It turns out that the results undergo at most only minor quantitative changes, and that the qualitative conclusions remain the same.

\section{Conclusion}

As discussed in the introduction, oceanic observations on internal solitary waves have brought to light a remarkable contrast between tropical regions, like the Sulu Sea, and the Bay of Biscay. First of all in their generation: in the Sulu Sea they stem from a disintegration of the internal tide which itself is generated by a direct forcing at the thermocline, in the Bay of Biscay they arise when an incoming internal-tide beam hits the thermocline.

In the latter case the waves thus forced at the thermocline would decay during their propagation even in the linear regime, because they consist of a number of modes and thus give rise to downward "leaking" (Gerkema, 2001). But, as is shown in the present study, in the nonlinear regime even the dominant (third) mode, taken in isolation, tends to de-

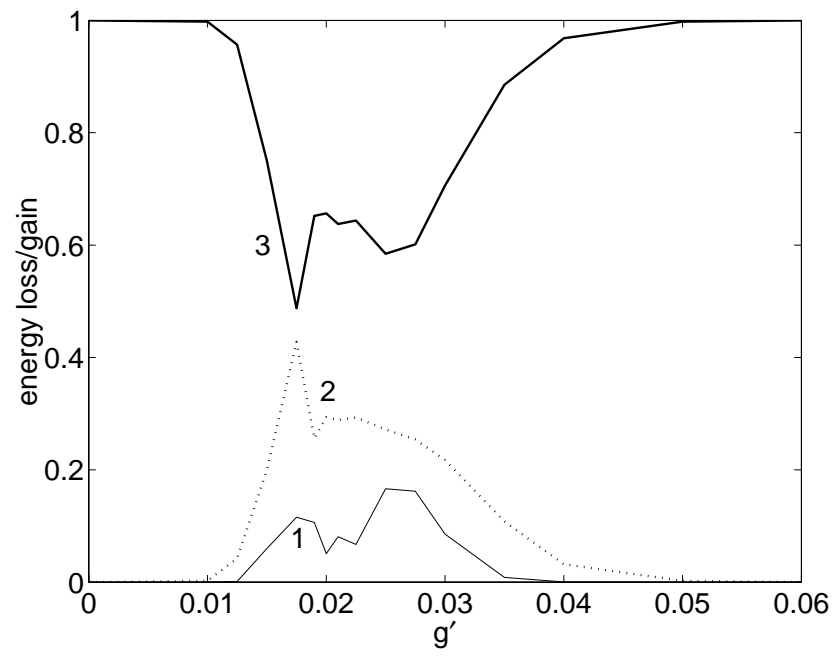

Fig. 11. The loss to the first two modes of the energy originally contained in the third-mode alone, as a function of the thermocline strength $g^{\prime}$.

cay, because it loses energy to other modes. As is clear from Fig. 11, this decay occurs for thermoclines of moderate strength - which is indeed the regime of the Bay of Biscay (in summer). By contrast, for strong thermoclines, the mode dominating the thermocline motion (the first mode) loses no significant amount of energy to other modes; this explains the persistence of the solitons observed in the Sulu Sea.

The different behaviour in different stratification regimes can be understood intuitively as follows. For a weak thermocline (regime A) the modal structures are nearly the same as for constant stratification, and hence interaction terms are weak (though not absent, see Eq. 6). For a strong thermocline (regime B), the first mode has an interfacial character while the other modes, which have a much lower phase speed, are similar to those of constant stratification (see $6 \mathrm{a}, \mathrm{b}$ : the second and third modes in $b$ have an overall similarity to the first and second modes in a); again one expects little energy exchange between modes. It is only for moderate thermoclines (regime $\mathrm{C}$ ) that a significant exchange is to be expected.

From a modelling point of view, the conclusion is that a uni-modal approach is not well suited for describing the evolution (i.e. generation and propagation) of internal solitary waves in the regime of moderate thermoclines (C), like in the Bay of Biscay. For strong thermoclines (tropical regions), by contrast, a uni-modal approach is justifiable. It should be emphasized that the terms "moderate" and "strong" are relative in the sense that they are weighed by the other factors making up the stratification; this is expressed by Eq. (19). The value of $g^{\prime}$, the thermocline strength as used in the 2c-layer system, can be obtained from empirical profiles by measuring the area enclosed by the $N^{2}$ versus $z$ graph (see Gerkema, 2001); hence not only the amplitude of $N$ plays a role but also the thermocline thickness. It is precisely the latter factor that creates the difference in thermocline regimes between the Sulu Sea and the Bay of Biscay (compare Figs. 1b, d). 
Two interesting features stand out in regime $\mathrm{C}$. The first one is the occurrence, in part of the regime, of persistent multi-modal twin-type solitary waves (Fig. 9), which deserve further (analytical) study. The second one is of interest in a wider oceanographic context. It is the co-occurrence of firstmode modulated wave groups which arise at the expense of the original third-mode depression (Figs. 9, 10). It underlines the fact that the thermocline region and abyssal ocean are closely connected dynamically, and that the occurrence of solitary waves near the thermocline may be accompanied by high-frequency wave trains in the deeper parts, and may thus affect at once the abyssal internal-wave spectrum.

Acknowledgements. The author thanks L. Maas for comments on an earlier version of the manuscript. This is NIOZ publication no. 3738 .

\section{References}

Apel, J. R.: Principles of ocean physics, Academic Press, 1987.

Apel, J. R., Holbrook, J. R., Liu, A. K., and Tsai, J. J.: The Sulu Sea internal soliton experiment, J. Phys. Oceanogr., 15, 1625-1651, 1985.

Baines, P. G.: On internal tide generation models, Deep-Sea Res., 29, 307-338, 1982.

Fletcher, C. A. J.: Computational techniques for fluid dynamics, Vol. 1, Springer-Verlag, 2nd edn., 1991.

Gerkema, T.: Nonlinear dispersive internal tides: generation models for a rotating ocean, $\mathrm{PhD}$ thesis, Utrecht University, 1994.

Gerkema, T.: A unified model for the generation and fission of internal tides in a rotating ocean, J. Mar. Res., 54, 421-450, 1996.

Gerkema, T.: Internal and interfacial tides: beam scattering and local generation of solitary waves, J. Mar. Res., 59, 227-255, 2001.

Gerkema, T. and Zimmerman, J. T. F.: Generation of nonlinear internal tides and solitary waves, J. Phys. Oceanogr., 25, 10811094, 1995.

Giese, G. S., Chapman, D. C., Collins, M. G., Encarnacion, R., and Jacinto, G.: The coupling between harbor seiches at Palawan Island and Sulu Sea internal solitons, J. Phys. Oc., 28, 2418 2426, 1998.

Lamb, K. G.: Numerical experiments of internal wave generation by strong tidal flow across a finite amplitude bank edge, J. Geophys. Res., 99, 843-864, 1994.

LeBlond, P. H. and Mysak, L. A.: Waves in the ocean, Elsevier, 1978.

Lee, C. and Beardsley, R. C.: The generation of long nonlinear internal waves in a weakly stratified shear flow, J. Geophys. Res., 79, 453-462, 1974.

Liu, A. K., Holbrook, J. R., and Apel, J. R.: Nonlinear internal wave evolution in the Sulu Sea, J. Phys. Oceanogr., 15, 16131624, 1985.

Maxworthy, T.: A note on the internal solitary waves produced by tidal flow over a three-dimensional ridge, J. Geophys. Res., 84, 338-346, 1979.

Miropol'sky, Y. Z.: Dynamics of internal gravity waves in the ocean, Kluwer, 2001.

New, A. L.: Internal tidal mixing in the Bay of Biscay, Deep-Sea Res., 35, 691-709, 1988.

New, A. L. and Da Silva, J. C. B.: Remote-sensing evidence for the local generation of internal soliton packets in the Central Bay of Biscay, Deep-Sea Res., 49, 915-934, 2002.

New, A. L. and Pingree, R. D.: Large-amplitude internal soliton packets in the central Bay of Biscay, Deep-Sea Res., 37, 513524, 1990.

New, A. L. and Pingree, R. D.: Local generation of internal soliton packets in the central Bay of Biscay, Deep-Sea Res., 39, 15211534, 1992.

New, A. L. and Pingree, R. D.: An intercomparison of internal solitary waves in the Bay of Biscay and resulting from Korteweg-de Vries-type theory, Progr. Oceanogr., 45, 1-38, 2000.

Osborne, A. R. and Burch, T. L.: Internal solitons in the Andaman Sea, Nature, 208, 451-460, 1980.

Ostrovsky, L. A.: Nonlinear internal waves in a rotating ocean, Oceanology, 18, 119-125, 1978.

Pingree, R. D. and New, A. L.: Abyssal penetration and bottom reflection of internal tide energy in the Bay of Biscay, J. Phys. Oceanogr., 21, 28-39, 1991.

Shrira, V. I.: On the propagation of a three-dimensional packet of weakly non-linear internal gravity waves, Int. J. Non-linear Mech., 16, 129-138, 1981.

Vlasenko, V. I.: Multimodal soliton of internal waves, Izvestiya, Atmospheric and oceanic physics, 30, 161-169, 1994.

Vlasenko, V. I. and Hutter, K.: Generation of second mode solitary waves by the interaction of a first mode soliton with a sill, Nonl. Proc. in Geophys., 8, 223-239, 2001. 